\title{
Acylphloroglucinols from the fern Elaphoglossum lindbergii
}

\author{
Cecilia Socolsky, ${ }^{a}$ Elena Cartagena, ${ }^{a}$ Yoshinori Asakawa, ${ }^{b}$ and Alicia Bardón ${ }^{\text {** }}$ \\ ${ }^{a}$ INQUINOA-CONICET, Ayacucho 471, Tucumán 4000, Argentina \\ ${ }^{b}$ Faculty of Pharmaceutical Sciences, Tokushima Bunri University, Yamashiro-cho, \\ Tokushima 770-8514, Japan \\ E-mail: alisan@fbaf.unt.edu.ar
}

\begin{abstract}
Four new acylphloroglucinols have been isolated from a diethyl ether extract of the rhizomes and roots of the fern Elaphoglossum lindbergii. Their structures were elucidated by extensive analysis of spectroscopic data and comparison with those previously reported for other Elaphoglossum and Dryopteris acylphloroglucinols. These compounds showed mild antibacterial activity and altered biofilm formation of the Gram (+) bacterium Staphylococcus aureus at 100 $\mu \mathrm{g} / \mathrm{mL}$.
\end{abstract}

Keywords: Acylphloroglucinols, Elaphoglossum lindbergii, antibacterial activity, biofilm formation

\section{Introduction}

In Argentina, the genus Elaphoglossum is represented by seven species: E. piloselloides (C. Presl) T. Moore, E. gayanum (Fée) T. Moore, E. yungense de la Sota, E. crassipes (Hieron.) Diels, E. lorentzii (Hieron.) H. Christ, E. lindbergii (Mett. ex Kuhn) Rosenst., and E. pachydermum (Fée) T. Moore. ${ }^{1}$ Our previous chemical studies on E. piloselloides, E. gayanum, and $E$. yungense showed that all of them contain acylphloroglucinols in their scales, rhizomes, and roots. ${ }^{2-4}$ In the last decades, attention has been drawn to these compounds due to their various biological activities, such as antidepressant, ${ }^{5}$ antibacterial, ${ }^{6}$ and molluscicidal. ${ }^{2}$ As part of our ongoing investigations on Elaphoglossum species, we examined the diethyl ether $\left(\mathrm{Et}_{2} \mathrm{O}\right)$ extract of E. lindbergii to isolate 4 new acylphloroglucinols. The antibacterial and antibiofilm activity of these compounds was evaluated against Staphylococcus aureus. 


\section{Results and Discussion}

Rhizomes and roots of $E$. lindbergii were air-dried, ground, and extracted with $\mathrm{Et}_{2} \mathrm{O}$. The extract was fractionated by $\mathrm{CC}$ on silica gel and the fraction containing acylphlorolgucinols (TLC detection) was further purified by normal phase HPLC to afford four new prenylated acylphloroglucinols that were named lindbergins A-D (1-4).
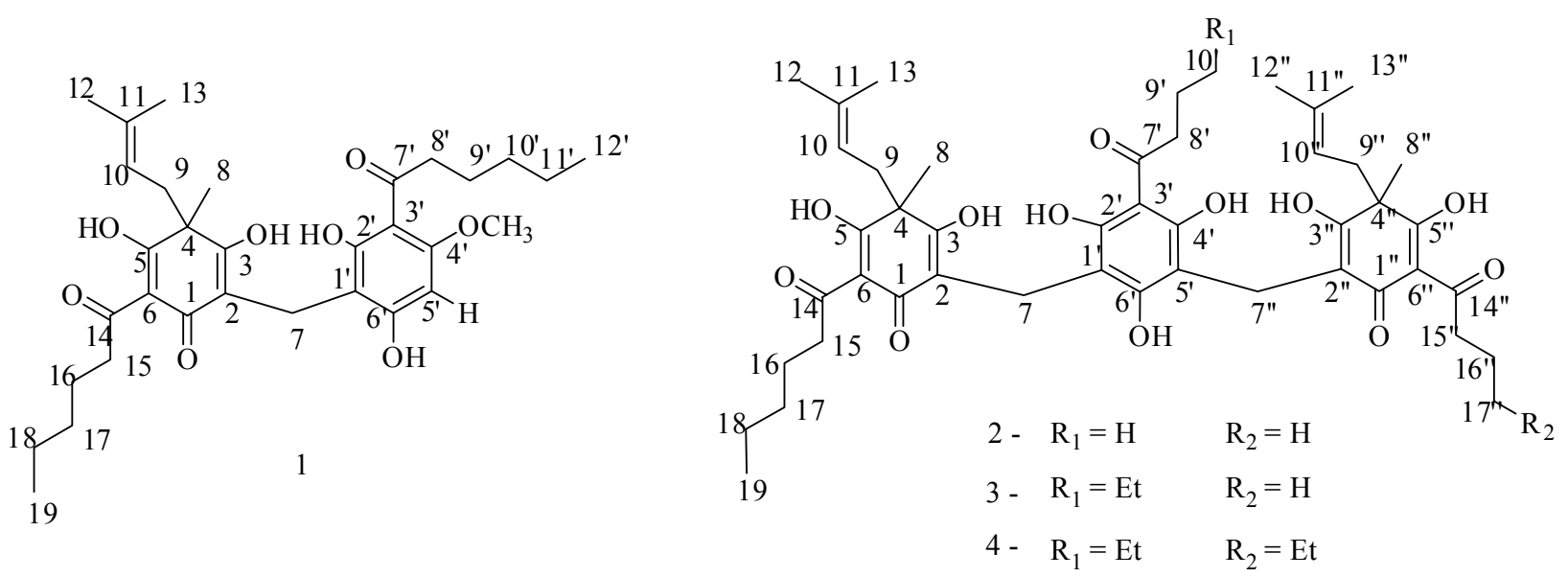

A high resolution measurement of the molecular ion of lindbergin A (1) gave $m / z$ 556.3043, pointing to the molecular formula $\mathrm{C}_{32} \mathrm{H}_{44} \mathrm{O}_{8}$ (calculated 556.3037). The ${ }^{1} \mathrm{H}$ and ${ }^{13} \mathrm{C}$ NMR spectra of 1 (Tables 1 and 2) resembled those of elaphogayanin B, previously isolated from $E$. gayanum. ${ }^{3}$ The NOESY spectrum of $\mathbf{1}$ showed crosspeaks between the $-\mathrm{OC}_{3}$ group and $\mathrm{H}-5^{\prime}$ and $\mathrm{H}-8^{\prime}$, revealing that the methoxy group was located at C-4' instead of at C-6' as in elaphogayanin B. Key H-C long range correlations of the hydroxyl protons, the methylene bridge protons, and $\mathrm{H}^{-5^{\prime}}$ with the ring carbons were observed in the $\mathrm{HMBC}$ spectrum of lindbergin $\mathrm{A}$ (1), allowing the unambiguous assignment of the ring carbons (Table 3). As previously observed for other phloroglucinol derivatives, ${ }^{2,3}$ the 5-OH hyroxyl proton shows HMBC correlations with $\mathrm{C}-14$ and $\mathrm{C}-15$, indicating that this proton is shared by the oxygen atoms at C-14 and C-5 forming a six-membered ring by hydrogen bonding. These crosspeaks are useful to identify the acyl group located at C-6 in acylphloroglucinol derivatives that possess an acylfilicinic acid-type ring. Full assignment of ${ }^{1} \mathrm{H}$ and ${ }^{13} \mathrm{C}$ spectra was accomplished by analysis of ${ }^{1} \mathrm{H}{ }^{1} \mathrm{H} \mathrm{COSY}$, HSQC and HMBC spectra. Based on the forementioned evidence, the structure of 1 was established as shown.

Lindbergin B (2) showed a molecular ion peak [M] $]^{+}$at $\mathrm{m} / \mathrm{z} 804.4091$ in its HRFABMS spectrum that was consistent with the molecular formula $\mathrm{C}_{46} \mathrm{H}_{60} \mathrm{O}_{12}$ (calculated 804.4086). An $\mathrm{AB}$ system signal accounting for the $2 \mathrm{CH}_{2}$ bridges was observed in the ${ }^{1} \mathrm{H}$ NMR spectrum of 2 centered at $\delta 3.56$, indicating that both bridges are attached to an aromatic and an acylfilicinic acid-type ring. ${ }^{7}$ For lindbergin $\mathrm{B}$, the ${ }^{1} \mathrm{H}$ NMR signals were broad, as previously observed for acylphloroglucinols containing more than two rings (Figure 1). ${ }^{7}$ The ${ }^{13} \mathrm{C}$ NMR spectrum showed 
duplicated or broad signals for the acylfilicinic acid-type ring carbons, suggesting the presence of two of such rings. Evidence for three acyl groups ( $\delta 209.6,207.8$, and 207.7) was obtained from the ${ }^{13} \mathrm{C}$ NMR spectrum of 2 (Table 2), confirming the presence of three rings, since in fern phloroglucinol derivatives, each ring only carries one acyl group. The acyl groups of $\mathbf{2}$ were identified as two butanoyls and one hexanoyl. It is worth pointing out that the methyl group of a hexanoyl residue is centered typically at $\delta 0.90-0.93$ in the ${ }^{1} \mathrm{H}$ NMR spectrum while that of a butanoyl moiety appears at lower field, $\delta 0.96-1.02 .^{2-4}$ This empirical observation can be useful to identify such residues in phloroglucinols containing three or more rings. The location of the acyl groups was established through HMBC correlations observed between 5-OH and both C-14 and C-14". From the above evidence, the structure of linbergin B (2) was deduced as depicted.

Table 1. ${ }^{1} \mathrm{H}$ NMR data of compounds $\mathbf{1 - 4}$ (acetone- $d_{6}, 500 \mathrm{MHz}$ )

\begin{tabular}{|c|c|c|c|c|}
\hline \multirow[b]{2}{*}{$H$} & \multicolumn{4}{|c|}{$\delta[\mathrm{ppm}]$, multiplicity, $J[\mathrm{~Hz}]$} \\
\hline & 1 & 2 & 3 & 4 \\
\hline $\mathrm{CH}_{2}-7$ & 3.53 & $3.56, J=16.5$ & $3.55, J=16.5$ & $3.55, J=16.8$ \\
\hline $\mathrm{CH}_{2}-7^{\prime \prime}$ & (center of AB system), $J$ & & & \\
\hline$(\mathrm{AB}$ & $=15.8$ & & & \\
\hline systems) & & & & \\
\hline $8,8^{\prime \prime}$ & $1.51, \mathrm{~s}$ & $1.53, \mathrm{~s}$ & $1.53, \mathrm{~s}$ & $1.53, \mathrm{~s}$ \\
\hline $9 a, 9^{a \prime \prime}$ & $2.71, \mathrm{dd}, J=13.5,9.0$ & $\begin{array}{l}2.73, \mathrm{dd}, \\
J=13.0,8.5\end{array}$ & $\begin{array}{l}2.73, \mathrm{dd}, \\
J=13.0,8.0\end{array}$ & $\begin{array}{l}2.72, \mathrm{dd}, \\
J=13.0,8.0\end{array}$ \\
\hline $9 b, 9 b^{\prime \prime}$ & $2.59, \mathrm{dd}, J=13.5,7.0$ & $2.69-2.61^{\mathrm{c}}$ & $2.68-2.60^{\mathrm{c}}$ & $2.68-2.60^{\mathrm{c}}$ \\
\hline $10,10^{\prime \prime}$ & $4.63, \mathrm{t}, J=7.0$ & 4.68 , br. s & 4.67 , br. s & 4.67 , br. s \\
\hline $12,12^{\prime \prime}$ & $1.32, \mathrm{~s}$ & $1.39, \mathrm{~s}$ & $1.38, \mathrm{~s}$ & $1.45-1.32^{\mathrm{a}}$ \\
\hline $13,13^{\prime \prime}$ & $1.38, \mathrm{~s}$ & $1.42, \mathrm{~s}$ & $1.40, \mathrm{~s}$ & $1.45-1.32^{\mathrm{a}}$ \\
\hline $15 \mathrm{a}$ & $\begin{array}{l}3.22, \mathrm{ddd}, J=15.0,8.5 \\
7.0\end{array}$ & $3.27-3.18^{\mathrm{a}}$ & $3.27-3.18^{\mathrm{a}}$ & $3.26-3.18^{\mathrm{a}}$ \\
\hline $15 b$ & $\begin{array}{l}3.10, \mathrm{ddd}, J=15.0,8.0 \\
7.0\end{array}$ & $3.15-3.06^{\mathrm{a}}$ & $3.05-2.95^{\mathrm{a}}$ & $3.12-3.05^{\mathrm{a}}$ \\
\hline 16 & $1.70-1.62^{\mathrm{a}}$ & $\begin{array}{l}\text { 1.67, quint., } \\
J=7.5\end{array}$ & $\begin{array}{l}\text { 1.66, quint., } \\
J=7.5\end{array}$ & $1.73-1.62^{\mathrm{a}}$ \\
\hline 17 & $1.40-1.34^{\mathrm{a}}$ & $1.46-1.34^{\mathrm{a}}$ & $1.43-1.32^{\mathrm{a}}$ & $1.45-1.32^{\mathrm{a}}$ \\
\hline 18 & $1.40-1.34^{\mathrm{a}}$ & $1.46-1.34^{\mathrm{a}}$ & $1.43-1.32^{\mathrm{a}}$ & $1.45-1.32^{\mathrm{a}}$ \\
\hline 19 & $0.91, \mathrm{t}, J=7.0^{\mathrm{b}}$ & $0.93, \mathrm{t}, J=7.0$ & $0.93, \mathrm{t}, J=6.5^{\mathrm{b}}$ & $0.93, \mathrm{t}, J=7.0$ \\
\hline $5^{\prime}$ & $6.12, \mathrm{~s}$ & - & - & - \\
\hline $8^{\prime}$ & $3.04, \mathrm{dt}, J=13.5,7.0$ & $3.25-3.13^{\mathrm{a}}$ & $3.25-3.09^{\mathrm{a}}$ & $3.23-3.08^{\mathrm{a}}$ \\
\hline $9^{\prime}$ & $1.70-1.62^{\mathrm{a}}$ & $1.74-1.63^{\mathrm{a}}$ & $\begin{array}{l}1.69, \text { quint., } J= \\
7.0\end{array}$ & $1.73-1.62^{\mathrm{a}}$ \\
\hline $10^{\prime}$ & $1.40-1.34^{\mathrm{a}}$ & $0.99, \mathrm{t}, J=7.5$ & $1.43-1.32^{\mathrm{a}}$ & $1.45-1.32^{\mathrm{a}}$ \\
\hline $11^{\prime}$ & $1.40-1.34^{\mathrm{a}}$ & - & $1.43-1.32^{\mathrm{a}}$ & $1.45-1.32^{\mathrm{a}}$ \\
\hline
\end{tabular}


Table 1. (Continued)

\begin{tabular}{lllll}
\hline $12^{\prime}$ & $0.92, \mathrm{t}, J=7.0^{\mathrm{b}}$ & - & $0.92, \mathrm{t}, J=7.0^{\mathrm{b}}$ & $0.92, \mathrm{t}, J=7.0$ \\
$15 \mathrm{a}^{\prime \prime}$ & - & $3.27-3.18^{\mathrm{a}}$ & $3.27-3.18^{\mathrm{a}}$ & $3.26-3.18^{\mathrm{a}}$ \\
$15 \mathrm{~b}^{\prime \prime}$ & - & $3.15-3.06^{\mathrm{a}}$ & $3.05-2.95^{\mathrm{a}}$ & $3.12-3.05^{\mathrm{a}}$ \\
$16^{\prime \prime}$ & - & $1.72, \mathrm{sext}$. & $1.73-1.63^{\mathrm{a}}$ & $1.73-1.62^{\mathrm{a}}$ \\
& & $J=7.0$ & & \\
$17^{\prime \prime}$ & - & $1.00, \mathrm{t}, J=7.0$ & $1.00, \mathrm{t}, J=7.5$ & $1.45-1.32^{\mathrm{a}}$ \\
$18^{\prime \prime}$ & - & - & - & $1.45-1.32^{\mathrm{a}}$ \\
$19^{\prime \prime}$ & - & - & - & $0.93, \mathrm{t}, J=7.0$ \\
$-\mathrm{OCH} H_{3}$ & $3.93, \mathrm{~s}$ & - & - & - \\
$3-\mathrm{OH}, 3^{\prime \prime}-$ & $9.92, \mathrm{~s}$ & $10.06, \mathrm{~s}$ & $10.06, \mathrm{~s}$ & $10.06, \mathrm{~s}$ \\
$\mathrm{OH}$ & & & & \\
$5-\mathrm{OH}, \quad 5^{\prime \prime}-$ & $18.66, \mathrm{~s}$ & $18.70, \mathrm{~s}$ & $18.70, \mathrm{~s}$ & $18.71, \mathrm{~s}$ \\
$\mathrm{OH}$ & & & & \\
$2^{\prime}-\mathrm{OH}$ & $16.60, \mathrm{~s}$ & $13.17, \mathrm{~s}$ & $13.17, \mathrm{~s}$ & $13.16, \mathrm{~s}$ \\
6 & $13.17, \mathrm{~s}$ & $13.17, \mathrm{~s}$ & $13.16, \mathrm{~s}$ \\
\hline
\end{tabular}

${ }^{\mathrm{a}}$ Overlapping signals, ${ }^{\mathrm{b}}$ Signals may be exchangeable, ${ }^{\mathrm{c}}$ Obscured.

Table 2. ${ }^{13} \mathrm{C}$ NMR data of compounds 1-4 (acetone- $d_{6}, 125 \mathrm{MHz}$ )

\begin{tabular}{ccccc}
\hline & \multicolumn{4}{c}{$\delta[\mathrm{ppm}]$, multiplicity } \\
\cline { 2 - 5 } $\mathrm{C}$ & $\mathbf{1}$ & $\mathbf{2}$ & $\mathbf{3}$ & $\mathbf{4}$ \\
\hline $1,1^{\prime \prime}$ & $189.5, \mathrm{qC}$ & $190.0, \mathrm{qC}$ & $190.0, \mathrm{qC}$ & $189.9, \mathrm{qC}$ \\
$2,2^{\prime \prime}$ & $115.2, \mathrm{qC}$ & $115.2, \mathrm{qC}$ & $115.2, \mathrm{qC}$ & $115.2, \mathrm{qC}$ \\
$3,3^{\prime \prime}$ & $171.7, \mathrm{qC}$ & $172.4, \mathrm{qC}$ & $172.5, \mathrm{qC}$ & $171.9, \mathrm{qC}$ \\
$4,4^{\prime \prime}$ & $50.7, \mathrm{qC}$ & $50.9, \mathrm{qC}$ & $50.9, \mathrm{qC}$ & $50.9, \mathrm{qC}$ \\
$5,5^{\prime \prime}$ & $200.0, \mathrm{qC}$ & $200.4, \mathrm{qC}$ & $200.4, \mathrm{qC}$ & $200.4, \mathrm{qC}$ \\
$6,6^{\prime \prime}$ & $111.7, \mathrm{qC}$ & $111.5, \mathrm{qC}$ & $111.5, \mathrm{qC}$ & $111.5, \mathrm{qC}$ \\
$7,7^{\prime \prime}$ & $17.8, \mathrm{CH}_{2}$ & $18.7, \mathrm{CH}_{2}$ & $18.7, \mathrm{CH}_{2}$ & $18.7, \mathrm{CH}_{2}$ \\
$8,8^{\prime \prime}$ & $24.2, \mathrm{CH}_{3}$ & $24.4, \mathrm{CH}_{3}$ & $24.5, \mathrm{CH}_{3}$ & $24.3, \mathrm{CH}_{3}$ \\
$9,9^{\prime \prime}$ & $40.0, \mathrm{CH}_{2}$ & $39.8, \mathrm{CH}_{2}$ & $39.8, \mathrm{CH}_{2}$ & $39.9, \mathrm{CH}_{2}$ \\
$10,10^{\prime \prime}$ & $119.3, \mathrm{CH}_{1}$ & $119.3, \mathrm{CH}^{\prime}$ & $119.3, \mathrm{CH}$ & $119.3, \mathrm{CH}$ \\
$11,11^{\prime \prime}$ & $137.4, \mathrm{qC}$ & $137.5, \mathrm{qC}$ & $137.5, \mathrm{qC}$ & $137.5, \mathrm{qC}$ \\
$12,12^{\prime \prime}$ & $26.6, \mathrm{CH}_{3}$ & $26.7, \mathrm{CH}_{3}$ & $26.7, \mathrm{CH}_{3}$ & $26.7, \mathrm{CH}_{3}$ \\
$13,13^{\prime \prime}$ & $18.6, \mathrm{CH}_{3}$ & $18.7, \mathrm{CH}_{3}$ & $18.7, \mathrm{CH}_{3}$ & $18.7, \mathrm{CH}_{3}$ \\
14 & $207.7, \mathrm{qC}$ & $207.7, \mathrm{qC}$ & $207.7^{a}, \mathrm{qC}$ & $207.7, \mathrm{qC}$ \\
15 & $42.3, \mathrm{CH}_{2}$ & $42.3, \mathrm{CH}_{2}$ & $42.3, \mathrm{CH}_{2}$ & $42.3, \mathrm{CH}_{2}$ \\
16 & $26.4, \mathrm{CH}_{2}$ & $26.5, \mathrm{CH}_{2}$ & $26.4, \mathrm{CH}_{2}$ & $26.4, \mathrm{CH}_{2}$ \\
17 & $33.3^{a}, \mathrm{CH}_{2}$ & $33.3, \mathrm{CH}_{2}$ & $33.3^{b}, \mathrm{CH}_{2}$ & $33.3^{a}, \mathrm{CH}_{2}$ \\
\hline
\end{tabular}


Table 2. (Continued)

\begin{tabular}{ccccc}
\hline 18 & $24.1, \mathrm{CH}_{2}$ & $24.1, \mathrm{CH}_{2}$ & $24.1^{c}, \mathrm{CH}_{2}$ & $24.1^{b}, \mathrm{CH}_{2}$ \\
19 & $15.3^{b}, \mathrm{CH}_{3}$ & $15.3, \mathrm{CH}_{3}$ & $15.3, \mathrm{CH}_{3}$ & $15.3, \mathrm{CH}_{3}$ \\
$1^{\prime}$ & $108.1, \mathrm{qC}$ & $108.0, \mathrm{qC}$ & $108.0, \mathrm{qC}$ & $108.0, \mathrm{qC}$ \\
$2^{\prime}$ & $164.9, \mathrm{qC}$ & $160.8, \mathrm{qC}$ & $160.7, \mathrm{qC}$ & $160.7, \mathrm{qC}$ \\
$3^{\prime}$ & $106.0, \mathrm{qC}$ & $107.2, \mathrm{qC}$ & $107.1, \mathrm{qC}$ & $107.1, \mathrm{qC}$ \\
$4^{\prime}$ & $164.2, \mathrm{qC}$ & $160.8, \mathrm{qC}$ & $160.7, \mathrm{qC}$ & $160.7, \mathrm{qC}$ \\
$5^{\prime}$ & $94.9, \mathrm{CH}$ & $108.0, \mathrm{qC}$ & $108.0, \mathrm{qC}$ & $108.0, \mathrm{qC}$ \\
$6^{\prime}$ & $166.2, \mathrm{qC}$ & $162.1, \mathrm{qC}$ & $162.2, \mathrm{qC}$ & $162.2, \mathrm{qC}$ \\
$7^{\prime}$ & $208.4, \mathrm{qC}$ & $209.6, \mathrm{qC}$ & $209.7, \mathrm{qC}$ & $209.7, \mathrm{qC}$ \\
$8^{\prime}$ & $45.2, \mathrm{CH}_{2}$ & $47.5, \mathrm{CH}_{2}$ & $45.5, \mathrm{CH}_{2}$ & $45.5, \mathrm{CH}_{2}$ \\
$9^{\prime}$ & $26.4, \mathrm{CH}_{2}$ & $20.0, \mathrm{CH}_{2}$ & $26.3, \mathrm{CH}_{2}$ & $26.2, \mathrm{CH}_{2}$ \\
$10^{\prime}$ & $33.4^{a}, \mathrm{CH}_{2}$ & $15.2^{a}, \mathrm{CH}_{3}$ & $33.4^{b}, \mathrm{CH}_{2}$ & $33.4^{a}, \mathrm{CH}_{2}$ \\
$11^{\prime}$ & $24.1, \mathrm{CH}_{2}$ & - & $24.2^{c}, \mathrm{CH}_{2}$ & $24.3^{b}, \mathrm{CH}_{2}$ \\
$12^{\prime}$ & $15.2^{b}, \mathrm{CH}_{3}$ & - & $15.3, \mathrm{CH}_{3}$ & $15.3, \mathrm{CH}_{3}$ \\
$14^{\prime \prime}$ & - & $207.8, \mathrm{qC}$ & $207.6^{a}, \mathrm{qC}$ & $207.7, \mathrm{qC}$ \\
$15^{\prime \prime}$ & - & $44.3, \mathrm{CH}_{2}$ & $44.3, \mathrm{CH}_{2}$ & $42.3, \mathrm{CH}_{2}$ \\
$16^{\prime \prime}$ & - & $19.8, \mathrm{CH}_{2}$ & $20.0, \mathrm{CH}_{2}$ & $26.4, \mathrm{CH}_{2}$ \\
$17^{\prime \prime}$ & - & $15.1^{a}, \mathrm{CH}_{3}$ & $15.2, \mathrm{CH}_{3}$ & $33.3^{a}, \mathrm{CH}_{2}$ \\
$18^{\prime \prime}$ & - & - & - & $24.1^{b}, \mathrm{CH}_{2}$ \\
$19^{\prime \prime}$ & - & - & - & $15.3, \mathrm{CH}_{3}$ \\
$-\mathrm{OCH}_{3}$ & $57.3, \mathrm{CH}_{3}$ & - & - & - \\
\hline
\end{tabular}

${ }^{\mathrm{a}-\mathrm{c}}$ Signals in a column marked with the same letter may be exchangeable.

The molecular formula of lindbergin $\mathrm{C}(3), \mathrm{C}_{48} \mathrm{H}_{64} \mathrm{O}_{12}$, was deduced from its HRFABMS (observed $m / z$ 832.4409, calculated 832.4399). Its 1D NMR data (Tables 1 and 2) resembled those of 2, but, in the ${ }^{1} \mathrm{H}$ NMR spectrum of compound 3 signals for one butanoyl and 2 hexanoyl residues were detected. The location of the acyl groups was established through long range $\mathrm{H}-\mathrm{C}$ correlations. Thus, the structure of lindbergin $\mathrm{C} \mathrm{(3)}$ was assessed as shown.

Lindbergin D (4) has a molecular formula $\mathrm{C}_{50} \mathrm{H}_{68} \mathrm{O}_{12}$ as deduced from a molecular ion peak at $\mathrm{m} / \mathrm{z} 860.4736$ in its HRFABMS spectrum (calculated 860.4712). The NMR traces of 4 resembled those of $\mathbf{2}$ and $\mathbf{3}$ but, in the case of $\mathbf{4}$, the presence of 3 hexanoyl residues was clearly observed. Accordingly, the structure of lindbergin D (4) was elucidated as illustrated.

The molecular formula of lindbergin $\mathrm{C} \mathrm{(3)}, \mathrm{C}_{48} \mathrm{H}_{64} \mathrm{O}_{12}$, was deduced from its HRFABMS (observed $\mathrm{m} / \mathrm{z}$ 832.4409, calculated 832.4399). Its 1D NMR data (Tables 1 and 2) resembled those of 2 , but, in the ${ }^{1} \mathrm{H}$ NMR spectrum of compound 3 signals for one butanoyl and 2 hexanoyl residues were detected. The location of the acyl groups was established through long range $\mathrm{H}-\mathrm{C}$ correlations. Thus, the structure of lindbergin $\mathrm{C} \mathrm{(3)}$ was assessed as shown. 
Table 3. Key H-C long range correlations for compounds 1-4

\begin{tabular}{|c|c|c|c|}
\hline \multicolumn{2}{|l|}{1} & \multicolumn{2}{|l|}{2} \\
\hline $\mathrm{H}$ & $\mathrm{C}$ & $\mathrm{H}$ & $\mathrm{C}$ \\
\hline 7 & $1,2,3,1^{\prime}, 2^{\prime}, 6^{\prime}$ & $7 / 7^{\prime \prime}$ & $1 / 1^{\prime \prime}, 2 / 2^{\prime \prime}, 3 / 3^{\prime \prime}, 1^{\prime} / 5^{\prime}, 2^{\prime} / 4^{\prime}, 6^{\prime}$ \\
\hline 8 & $3,4,5,9,10$ & $8 / 8^{\prime \prime}$ & $3 / 3^{\prime \prime}, 4 / 4^{\prime \prime}, 5 / 5^{\prime \prime}, 9 / 9^{\prime \prime}, 10 / 10^{\prime \prime}$ \\
\hline $9 a, 9 b$ & $3,4,5,8,10,11$ & $9 \mathrm{a} / 9 \mathrm{a}^{\prime \prime}$ & $4 / 4^{\prime \prime}, 5 / 5^{\prime \prime}, 10 / 10^{\prime \prime}, 11 / 11^{\prime \prime}$ \\
\hline 10 & $9,12,13$ & $12 / 12^{\prime \prime}$ & $10 / 10^{\prime \prime}, 11 / 11^{\prime \prime}, 13 / 13^{\prime \prime}$ \\
\hline 12 & $10,11,13$ & $13 / 13^{\prime \prime}$ & $12 / 12^{\prime \prime}$ \\
\hline $15 a, 15 b$ & $14,16,17$ & $15 a, 15 b$ & $14,16,17$ \\
\hline $5^{\prime}$ & $1^{\prime}, 3^{\prime}, 4^{\prime}, 6^{\prime}, 7^{\prime}$ & 16 & 17,18 \\
\hline $8^{\prime}$ & $7^{\prime}, 9^{\prime}, 10^{\prime}$ & 19 & 17,18 \\
\hline$-\mathrm{OC}_{3}$ & $4^{\prime}, 5^{\prime}$ & $8^{\prime}$ & $7^{\prime}$ \\
\hline $3-\mathrm{OH}$ & 4 & $9^{\prime}$ & $7^{\prime}, 8^{\prime}$ \\
\hline $5-\mathrm{OH}$ & $4,5,6,14,15$ & $3-\mathrm{OH} / 3^{\prime \prime}-\mathrm{OH}$ & $4 / 4^{\prime \prime}$ \\
\hline $2^{\prime}-\mathrm{OH}$ & $1^{\prime}, 2^{\prime}, 3^{\prime}$ & $5-\mathrm{OH} / 5^{\prime \prime}-\mathrm{OH}$ & $4 / 4^{\prime \prime}, 5 / 5^{\prime \prime}, 6 / 6^{\prime \prime}, 14 / 14^{\prime \prime}$ \\
\hline \multirow[t]{3}{*}{$6^{\prime}-\mathrm{OH}$} & $1^{\prime}, 5^{\prime}, 6^{\prime}$ & $2^{\prime}-\mathrm{OH}$ & $2^{\prime}$ \\
\hline & & $15 \mathrm{a}^{\prime \prime}$ & $14^{\prime \prime}$ \\
\hline & & $17^{\prime \prime}$ & $15^{\prime \prime}, 16^{\prime \prime}$ \\
\hline 3 & & 4 & \\
\hline $\mathrm{H}$ & $\mathrm{C}$ & $\mathrm{H}$ & $\mathrm{C}$ \\
\hline $7 / 7^{\prime \prime}$ & $1 / 1^{\prime \prime}, 2 / 2^{\prime \prime}, 3 / 3^{\prime \prime}, 1^{\prime} / 5^{\prime}, 2^{\prime} / 4^{\prime}, 6^{\prime}$ & $7 / 7^{\prime \prime}$ & $1 / 1^{\prime \prime}, 2 / 2^{\prime \prime}, 3 / 3^{\prime \prime}, 1^{\prime} / 5^{\prime}, 2^{\prime} / 4^{\prime}, 6^{\prime}$ \\
\hline $8 / 8^{\prime \prime}$ & $3 / 3^{\prime \prime}, 4 / 4^{\prime \prime}, 5 / 5^{\prime \prime}, 9 / 9^{\prime \prime}$ & $8 / 8^{\prime \prime}$ & $3 / 3^{\prime \prime}, 4 / 4^{\prime \prime}, 5 / 5^{\prime \prime}, 9 / 9^{\prime \prime}$ \\
\hline $9 \mathrm{a} / 9 \mathrm{a}^{\prime \prime}$ & $4 / 4^{\prime \prime}, 5 / 5^{\prime \prime}, 10 / 10^{\prime \prime}, 11 / 11^{\prime \prime}$ & $9 \mathrm{a} / 9 \mathrm{a}^{\prime \prime}$ & $4 / 4^{\prime \prime}, 5 / 5^{\prime \prime}, 10 / 10^{\prime \prime}, 11 / 11^{\prime \prime}$ \\
\hline $12 / 12^{\prime \prime}$ & 10/10", 11/11", 13/13" & $12 / 12^{\prime \prime}$ & $10 / 10^{\prime \prime}, 11 / 11^{\prime \prime}$ \\
\hline $13 / 13^{\prime \prime}$ & $10 / 10^{\prime \prime}, 11 / 11^{\prime \prime}, 12 / 12^{\prime \prime}$ & $13 / 13^{\prime \prime}$ & $10 / 10^{\prime \prime}, 11 / 11^{\prime \prime}$ \\
\hline $15 \mathrm{a}$ & $14,16,17$ & $15 \mathrm{a} / 15 \mathrm{a}^{\prime \prime}$ & 14/14", 16/16", 17/17" \\
\hline $8^{\prime}$ & $7^{\prime}$ & $16 / 16^{\prime \prime}$ & 14/14", 17/17"', 18/18" \\
\hline $3-\mathrm{OH} / 3^{\prime \prime}-\mathrm{OH}$ & $2 / 2^{\prime \prime}, 4 / 4^{\prime \prime}$ & $8^{\prime}$ & $7^{\prime}, 9^{\prime}, 10^{\prime}$ \\
\hline $5-\mathrm{OH} / 5^{\prime \prime}-\mathrm{OH}$ & $4 / 4^{\prime \prime}, 5 / 5^{\prime \prime}, 6 / 6^{\prime \prime}, 14 / 14^{\prime \prime}$ & $9^{\prime}$ & $7^{\prime}, 10^{\prime}, 11^{\prime}$ \\
\hline $2^{\prime}-\mathrm{OH}$ & $1^{\prime}, 2^{\prime}, 3^{\prime}$ & $3-\mathrm{OH} / 3^{\prime \prime}-\mathrm{OH}$ & $4 / 4^{\prime \prime}$ \\
\hline $15^{\prime \prime}$ & $14^{\prime \prime}, 16^{\prime \prime}$ & $5-\mathrm{OH} / 5^{\prime \prime}-\mathrm{OH}$ & $4 / 4^{\prime \prime}, 5 / 5^{\prime \prime}, 6 / 6^{\prime \prime}, 14 / 14^{\prime \prime}$ \\
\hline $17^{\prime \prime}$ & $15^{\prime \prime}, 16^{\prime \prime}$ & $2^{\prime}-\mathrm{OH}$ & $2^{\prime}, 3^{\prime}$ \\
\hline
\end{tabular}

Lindbergin D (4) has a molecular formula $\mathrm{C}_{50} \mathrm{H}_{68} \mathrm{O}_{12}$ as deduced from a molecular ion peak at $m / z 860.4736$ in its HRFABMS spectrum (calculated 860.4712). The NMR traces of 4 resembled those of $\mathbf{2}$ and $\mathbf{3}$ but, in the case of $\mathbf{4}$, the presence of 3 hexanoyl residues was clearly observed. Accordingly, the structure of lindbergin D (4) was elucidated as illustrated. 

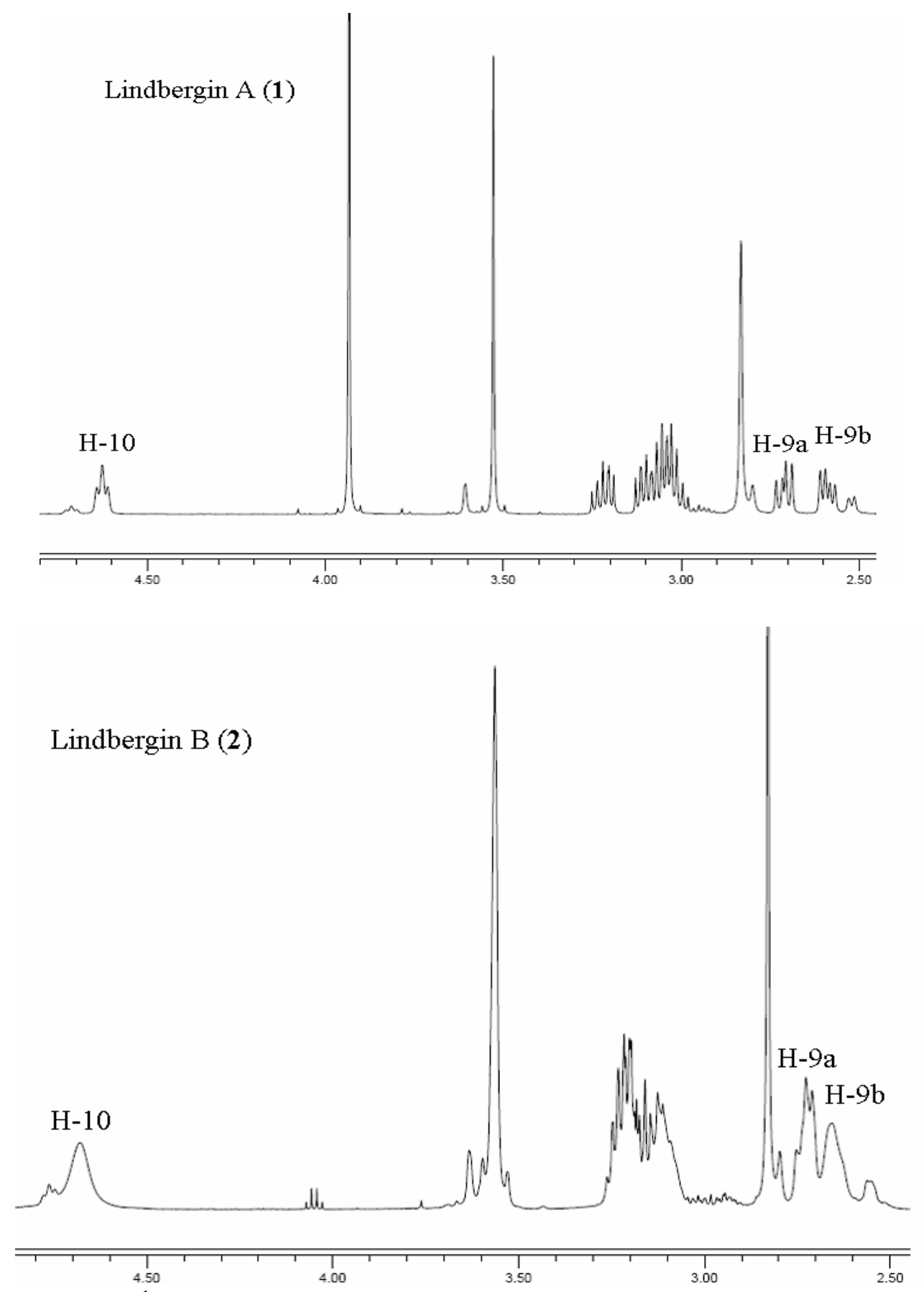

Figure 1. Part of the ${ }^{1} \mathrm{H}$ NMR spectra of compounds 1 and $2\left(500 \mathrm{MHz}\right.$, acetone- $\left.d_{6}\right)$.*

* Note that for lindbergin B (2), containing three rings, some ${ }^{1} \mathrm{H}$ NMR signals are broadened in comparison with those of lindbergin A (1) and their multiplicity is almost completely undetected.

\section{Effects on biofilm}

Biofilms, which are aggregations of sessile bacteria surrounded by a polymeric matrix, ${ }^{8}$ are the leading cause of chronic nosocomial infections. Diseases such as endocarditis, osteomyelitis and medical device-related infections are caused by $S$. aureus biofilms and are not readily treatable 
with antibiotics. In fact, biofilms are resistant to antibiotic levels 10 up to 1,000 -fold higher than planktonic or free-floating bacteria. ${ }^{8}$ The aim of this research was to determine the effects of compounds $1-4$ on $S$. aureus biofilm and to analyze their mode of action.

Lindbergin B (2) considerably inhibited biofilm production by S. aureus (82\% at 100 $\mu \mathrm{g} / \mathrm{mL}$, Figure 2). At lower concentrations $(50,25$, and $12.5 \mu \mathrm{g} / \mathrm{mL})$ no significant difference with the control was detected. As the reduction in bacterial growth was $86 \%$ at the mentioned dose, apparently, the observed inhibition in biofilm formation might simply be a consequence of reduced growth. It is important to point out that the antibiotic azithromycin (positive control) produced $76 \%$ reduction of biofilm at $25 \mu \mathrm{g} / \mathrm{mL}$. Acylphloroglucinols affect bacterial growth and biofilm formation in different ways. While lindbergin B (2) reduced both, yungensins A, B, and D-F, previously isolated from E. yungense, ${ }^{4}$ stimulated biofilm production but reduced bacterial growth.

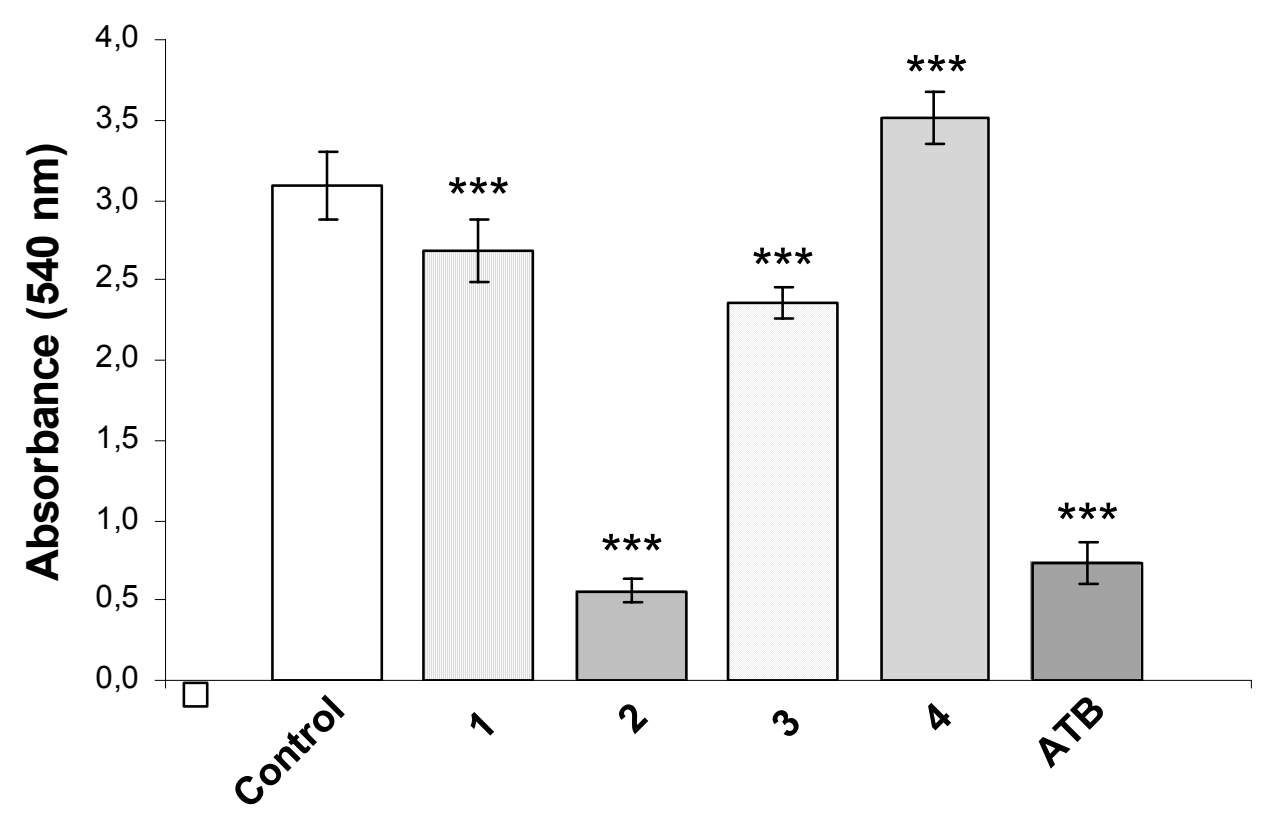

ATB: azithromycin.

*** Significant differences in mean values compared to control (Tuckey test).

Figure 2. Effect of $100 \mu \mathrm{g} / \mathrm{mL}$ of compunds 1-4 on biofilm production by $S$. aureus ATCC 6538 $\mathrm{P}$ evaluated through absorbance measurements $(540 \mathrm{~nm})$ employing the crystal violet staining method.

It is believed that lipophilic compounds intercalate in bacterial membranes, altering their fluidity. ${ }^{9}$ Bacteria then produce a hydrophilic exopolysaccharide that constitutes a physical barrier to the intercalation, as an adaptation strategy to a hostile environment. ${ }^{9}$ This phenomenon might be useful to explain the stimulation of biofilm formation produced by compound 4 (14 \%), 
that carries several lipophilic moieties, as well at that of yungensins A, B, and D-F, ${ }^{4}$ that carry one or two geranyl groups. The intercalation of these compounds inside the membrane would greatly alter membrane fluidity leading to cell lysis.

In addition, lindbergins $\mathrm{A}-\mathrm{D}$, produced no significant disruption of mature biofilm compared to control at 50,25 , and $12.5 \mu \mathrm{g} / \mathrm{mL}$.

\section{Antibacterial activity}

Acylphloroglucinols 1-4 show mild antibacterial acitivity against an ATCC collection strain of $S$. aureus with MICs $>100 \mu \mathrm{g} / \mathrm{mL}$ in all cases. At $100 \mu \mathrm{g} / \mathrm{mL}, 45,86,41$ and $35 \%$ growth inhibitions were detected after $24 \mathrm{~h}$ incubation, respectively. It is noteworthy that compound 2 strongly inhibited bacterial growth in the first $11 \mathrm{~h}$ of incubation $(\mathrm{OD}<0.05)$.

\section{Experimental Section}

General. Optical rotations were measured on a JASCO P-1030 polarimeter. IR spectra were recorded by the diffuse reflectance method on a Shimadzu FT/IR-8400S spectrophotometer. Low and high resolution mass spectra were registered in the positive mode on a JEOL JMS AX-500 spectrometer. 1 and 2D NMR experiments were performed with standard pulse sequences and parameters on a Varian Unity 500 using acetone- $d_{6}$ as solvent and internal reference. Column chromatography (CC) was carried out over silica gel (70-230 mesh) with an $n$-hexane-EtOAc gradient as eluent. Preparative high pressure liquid chromatography (HPLC) was performed on a Gilson instrument equipped with a Chemcopak silica gel column (Chemcosorb 5 Si-U, $5 \mu \mathrm{m}, 250$ $\times 10 \mathrm{~mm}$ i.d.). Thin layer chromatography was carried out on glass sheets coated with silica gel $60 \mathrm{~F}_{254}$ (Merck) and detection was accomplished under UV light and further spraying with Godin reagent ${ }^{10}$ followed by heating on a hot plate.

Plant material. E. lindbergii was collected on Route 29, Tiraxi, Jujuy province (GPS: $24^{\circ} 00^{\prime} 57^{\prime \prime}$ S, 65 $23^{\prime} 33^{\prime \prime} \mathrm{W}$ ), and identified by Marcela Hernández de Terán. A voucher specimen (LIL 609963) was deposited at Herbarium of Fundación Miguel Lillo, Tucumán, Argentina.

Extraction and isolation. Powdered rhizomes and roots of E. lindbergii $(59 \mathrm{~g})$ were extracted twice with $\mathrm{Et}_{2} \mathrm{O}$ at room temperature. Further filtration and evaporation of the combined extracts afforded $2.3 \mathrm{~g}$ of a dark red gum. Column chromatography of the crude extract over $\mathrm{SiO}_{2}$ gave one acylphloroglucinol-containing fraction $(956.2 \mathrm{mg})$. Processing of a portion of this sample $(220 \mathrm{mg}$ ) by normal phase HPLC ( $n$-hexane-EtOAc $98.2,3.5 \mathrm{~mL} / \mathrm{min}$ ) yielded 3 major fractions. Fraction $1(64.2 \mathrm{mg})$ gave $4(49 \mathrm{mg})$ upon HPLC purification (n-hexane-EtOAc 99.5:0.5, 0.2\% HOAc, $4.0 \mathrm{~mL} / \mathrm{min})$. Further chromatography of fraction $2(29.4 \mathrm{mg}$ ) by NPHPLC ( $n$-hexaneEtOAc 99:1, 0.4\% HOAc, $4.0 \mathrm{~mL} / \mathrm{min}$ ) furnished $3(25.7 \mathrm{mg})$. Processing of fraction $3(34.9 \mathrm{mg})$ under the same conditions yielded $\mathbf{1}(10.3 \mathrm{mg})$ and $\mathbf{2}(10.1 \mathrm{mg})$. 
Lindbergin A (1). yellow oil, $[\alpha]^{21.8}-49.8\left(c\right.$ 1.0, $\left.\mathrm{CHCl}_{3}\right)$. IR (neat, $\left.\mathrm{cm}^{-1}\right) v_{\max }: 3165,2721$, 2660, 1641, 1595. ${ }^{1} \mathrm{H}$ NMR data $\left(500 \mathrm{MHz}\right.$, acetone- $\left.d_{6}\right)$ in Table $1 .{ }^{13} \mathrm{C}$ NMR data $(125 \mathrm{MHz}$, acetone- $d_{6}$ ) in Table 2. HREIMS, $\mathrm{m} / \mathrm{z}: 556.3043$ (calculated for $\mathrm{C}_{32} \mathrm{H}_{44} \mathrm{O}_{8}: 556.3037$ ).

Lindbergin B (2). yellow gum, $[\alpha]_{D}^{22.6} 0\left(c 1.0, \mathrm{CHCl}_{3}\right)$. IR (neat, $\left.\mathrm{cm}^{-1}\right) v_{\max }: 3157,2723,2644$, $2608,1637,1610,1543 .{ }^{1} \mathrm{H}$ NMR data $\left(500 \mathrm{MHz}\right.$, acetone- $\left.d_{6}\right)$ in Table $1 .{ }^{13} \mathrm{C}$ NMR data $(125$ MHz, acetone- $d_{6}$ ) in Table 2. HRFABMS, m/z: 804.4091 (calculated for $\mathrm{C}_{46} \mathrm{H}_{60} \mathrm{O}_{12}:$ 804.4086). Lindbergin $\mathrm{C}$ (3). yellow gum, $[\alpha]^{21.9}-1.8\left(c 1.0, \mathrm{CHCl}_{3}\right)$. IR (neat, $\left.\mathrm{cm}^{-1}\right) v_{\max }: 3153,2640$, $2608,1637,1612,1551 .{ }^{1} \mathrm{H}$ NMR data $\left(500 \mathrm{MHz}\right.$, acetone- $\left.d_{6}\right)$ in Table $1 .{ }^{13} \mathrm{C}$ NMR data $(125$ MHz, acetone- $d_{6}$ ) in Table 2. HRFABMS, m/z: 832.4409 (calculated for $\mathrm{C}_{48} \mathrm{H}_{64} \mathrm{O}_{12}: 832.4399$ ). Lindbergin D (4). yellow gum, $[\alpha]_{\mathrm{D}}^{21.9}-1.9\left(c 1.0, \mathrm{CHCl}_{3}\right.$ ). IR (neat, $\left.\mathrm{cm}^{-1}\right) v_{\max }: 3155,2629$, $2604,1637,1612,1549 .{ }^{1} \mathrm{H}$ NMR data $\left(500 \mathrm{MHz}\right.$, acetone- $\left.d_{6}\right)$ in Table $1 .{ }^{13} \mathrm{C}$ NMR data $(125$ $\mathrm{MHz}$, acetone- $d_{6}$ ) in Table 2. HRFABMS, m/z: 860.4736 (calculated for $\mathrm{C}_{50} \mathrm{H}_{68} \mathrm{O}_{12}: 860.4712$ ).

Antibacterial activity. The test was performed in sterile 96-well microplates. Different concentrations of the samples $(100,50,25,12.5$, and $6.2 \mu \mathrm{g} / \mathrm{mL})$ were tested against $S$. aureus ATCC $6538 \mathrm{P}$. The inoculum $(180 \mu \mathrm{L})$ contained $1 \times 10^{6} \mathrm{CFU}$ approximately. Mueller-Hinton (MH) medium was employed to prepare the inoculum and the dilutions of the samples. The control contained the solvent mixture (EtOH-EtOAc) used to dissolve the samples (the final concentrations of EtOH and EtOAc did not exceed $0.7 \%$ and $0.3 \%$, respectively). Plates were incubated at $37{ }^{\circ} \mathrm{C}$ during $24 \mathrm{~h}$. Growth was detected as turbidity $(600 \mathrm{~nm})$ relative to its record at the beginning of the experiment. Measurements were performed using a microtitre plate reader (Power Wave XS2, Biotek, Vermont, USA). The assay was carried out in eight replicates for each compound. Azithromycin $(25 \mu \mathrm{g} / \mathrm{mL})$ was used as positive control.

Biofilm experiments. ${ }^{11}$ The effect of compounds 1-4 on biofilm production by S. aureus ATCC $6538 \mathrm{P}$ was evaluated at $100,50,25$, and $12.5 \mu \mathrm{g} / \mathrm{mL}$. The test was performed by the crystal violet staining method. After $24 \mathrm{~h}$ of incubation of the strain in the presence of the evaluated compound, the amount of biofilm was measured by staining bound cells with crystal violet (1\% w/v) for $20 \mathrm{~min}$. Then, the medium was discarded, the wells were rinsed with $\mathrm{H}_{2} \mathrm{O}$ (x2), the dye was dissolved in $200 \mu \mathrm{L}$ of EtOH, and absorbance was recorded at $540 \mathrm{~nm}$ using a microtitre plate reader. Eight replicates were performed for each sample.

Disruption of mature biofilm was evaluated after two hours of incubation in presence of the tested compounds (50,25 and $12.5 \mu \mathrm{g} / \mathrm{mL}$ ). An overnight culture of $S$. aureus, $20 \mu \mathrm{L}$, was placed on each well. A dilution of the sample to be tested in the culture medium (MH) was added to reach a final volume of $200 \mu \mathrm{L}$. After $2 \mathrm{~h}$, the biofilm was quantified as described in the previous paragraph. 


\section{Acknowledgements}

Authors thank Marcela Hernández de Terán for the identification of the plant material and Miss Y. Okamoto for the measurement of MS. C. Socolsky thanks the Ministry of Education, Culture, Sports, Science, and Technology of Japan for a fellowship. This work was partially supported by ANPCyT, CONICET and CIUNT, Argentina.

\section{References}

1. Lavalle, M. C.; Rodríguez, M. Darwiniana 2009, 47, 125.

2. Socolsky, C.; Borkosky, S. A.; Asakawa, Y.; Bardón, A. J. Nat. Prod. 2009, 72, 787.

3. Socolsky, C.; Borkosky, S. A.; Hernández de Terán, M.; Asakawa, Y.; Bardón, A. J. Nat. Prod. 2010, 73, 901.

4. Socolsky, C.; Arena, M. E.; Bardón, A. J. Nat. Prod. 2010, 73, 1751.

5. Di Carlo, G.; Borrelli, F.; Ernst, E.; Izzo, A. A. Trends Pharmacol. Sci. 2001, 22, 292.

6. Lee, H. B.; Kim, J. Ch.; Lee, S. M. Arch. Pharm. Res. 2009, 32, 655.

7. V. Euw, J.; Reichstein, T.; Widén, C.-J. Helv. Chim. Acta 1985, 68, 1251.

8. Monroe, D. PLoS Biol. 2007, 5, 2458.

9. Van Hamme, J. D; Singh, A.; Ward, O. P. Microbiol. Mol. Biol. Rev. 2003, 67, 503.

10. Godin, P. Nature 1954, 174, 134.

11. O’Toole, G. A.; Kolter, R. Mol. Microbiol. 1998, 28, 449. 